\title{
Modelling the evolution of solar-mass stars with a range of metallicities using MESA
}

\author{
E.F. Jones ${ }^{\mathrm{a}}$ and P.M. Gore \\ FM Research Professionals, P.O. Box 58412, Nashville, TN 37205 USA
}

\begin{abstract}
The nuclides ${ }^{1,2} \mathrm{H},{ }^{3,4} \mathrm{He},{ }^{7} \mathrm{Li},{ }^{7} \mathrm{Be},{ }^{8} \mathrm{~B},{ }^{12,13} \mathrm{C},{ }^{13-15} \mathrm{~N},{ }^{14-18} \mathrm{O},{ }^{17-19} \mathrm{~F},{ }^{18-22} \mathrm{Ne},{ }^{22} \mathrm{Mg}$, and ${ }^{24} \mathrm{Mg}$ were used in the code package MESA (Modules for Experiments in Stellar Astrophysics)[Paxton] to model a one-solarmass star with a range of metallicities, $z$, from 0 to 0.1 . On HR diagrams of each star model's luminosity and effective temperature from before zero-age main sequence (pre-ZAMS) to white dwarf, oscillations were noted in the horizontal branch at intervals from $z=0$ to 0.0070 . At $z=0$, the calculated stellar lifetime is $6.09 \times 10^{9}$ years. The calculated lifetime of the model stars increases to a maximum of $1.25 \times 10^{10}$ years at $z=0.022$ and then decreases to $2.59 \times 10^{9}$ years at $z=0.1$. A piecewise fit of the model lifetimes vs. metallicity was obtained.
\end{abstract}

Using the star module within the code package MESA (Modules for Experiments in Stellar Astrophysics) [1], a one-solar-mass star was modelled with 44 values of the metallicity ranging from $z=0$ to $z=0.1$ where $z$ is defined as the mass fraction of elements with atomic number greater than that of helium. The initial star mass was set to one solar mass, and the pre-main-sequence to white dwarf scenario from the test suites within the star module and the pp_cno_extras_o18_ne22 reaction network were used. The nuclides included in our MESA models were ${ }^{1,2} \mathrm{H},{ }^{3,4} \mathrm{He},{ }^{7} \mathrm{Li},{ }^{7} \mathrm{Be},{ }^{8} \mathrm{~B},{ }^{12,13} \mathrm{C},{ }^{13-15} \mathrm{~N},{ }^{14-18} \mathrm{O}$, ${ }^{17-19} \mathrm{~F},{ }^{18-22} \mathrm{Ne},{ }^{22} \mathrm{Mg}$, and ${ }^{24} \mathrm{Mg}$.

From HR diagrams of the luminosity and effective temperature of the star models from before zero-age main sequence (pre-ZAMS) to white dwarf, it was noted that some tracks exhibited one or more thermal pulses and retreats along the horizontal branch before finally turning down in the HR diagram to become a white dwarf. These oscillations occur in the horizontal branch for $z=0$ to 0.0004 , and then are not present for $z=0.0005$ through $z=0.0007$. Oscillations in the horizontal branch resume at $z=0.0008$ and become more pronounced until $z=0.002$. Here, the oscillations in the horizontal branch cease until their return for $z=0.006$ and $z=0.007$. Three simulations, for $z=0.003,0.004$, and 0.005 , climb to an aphysical state with high luminosity and relatively low temperature instead of reaching a white dwarf stage.

A piece-wise least-squares fit of the stellar lifetime as a function of metallicity was obtained. The piecewise fitting function is shown in Equation 1 where $t$ is the model lifetime in billions of years and $z$ is the metallicity as previously defined. The RMS errors of the segments of the fit in billions of years were $0.008,0.003,0.009$, and 0.009 , respectively.

$$
t=\left\{\begin{array}{c}
225.800 z^{0.864537}+5.889 \\
0 \leq z<0.01 \\
349.162 z^{1.125}+8.1830 \\
0.01 \leq z<0.02 \\
12.40-31519(z-0.02123)^{2}+6.545 z \\
0.02 \leq z<0.03 \\
18.344 e^{-16.108 z}-1.092 \\
0.03 \leq z \leq 0.1
\end{array}\right\}
$$

Our formula can be compared to Hurley et al. [2], which expressed the time to the base of the giant branch as a function of $z$ and $M$, but was fitted for a range of twenty-five values of $M$ and seven values of $z$ ranging from 0.0001 to 0.03 . For $M=1$ solar mass and defining $\zeta$ $=\log (z / 0.02)$, the formula for the time to the base of the giant branch from reference [3] reduces to the following:

$$
t_{B G B}=\frac{6.766741+2.487727 \zeta+0.208575 \zeta^{2}+8.347888 \zeta^{3}}{7.568309+4.564888 \zeta+2.958542 \zeta^{2}+5.571483 \zeta^{3}}
$$

In Equation 2, $t_{B G B}$ has a value of zero for $z=$ 0.00293299 and is undefined for $z=0.00188873$ which yielded a discontinuity when applied to the range of our 44 values of $z$.

A plot of model solar mass star lifetime vs. initial metallicity is shown in Figure 1. The calculated stellar lifetime for $z=0$ is $6.09 \times 10^{9}$ years. The lifetime of the model stars increases to a maximum of $1.25 \times 10^{10}$ at $z=0.022$ and then decreases to $2.59 \times 10^{9}$ years for $z=0.1$. A recent estimate [3] of the solar metallicity derived from pre-solar meteoritic grains and 3D

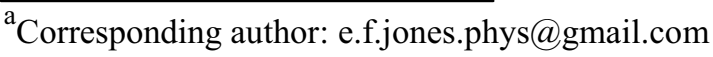


modelling is $z=0.0122$. Our model using this initial value of metallicity yielded a stellar lifetime of 10.63 billion years.

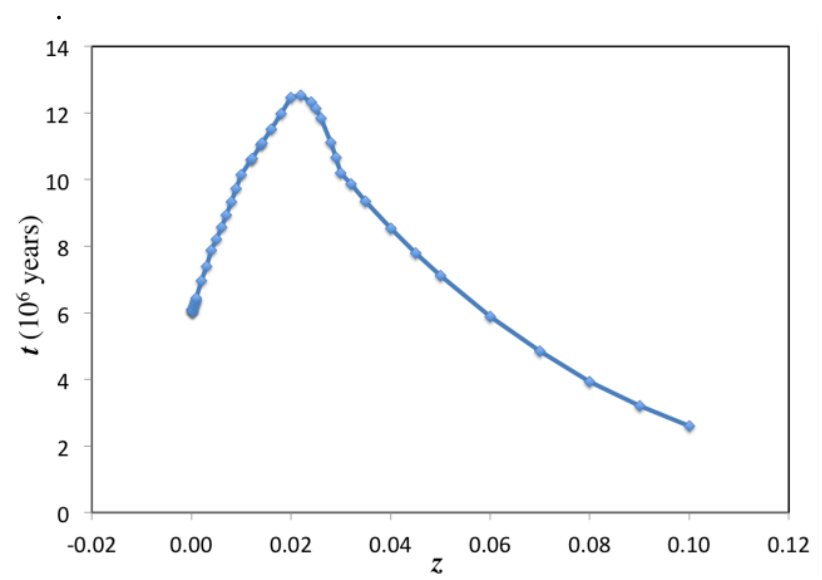

Figure 1. Model lifetime $t$ in billions of years vs metallicity $z$

The HR diagram showing the track of our simulation of a 1 solar mass star with metallicity $z=0.0122$ is shown below in Figure 2 in which pulses in the horizontal branch are evident:

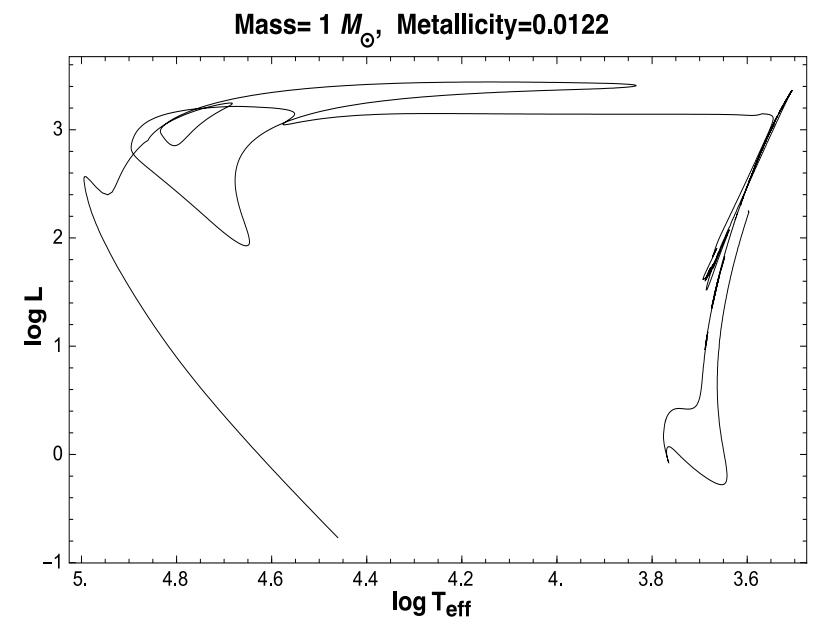

Figure 2. HR diagram of track from our simulation of a 1 solar mass star with $z=0.0122$.

Paxton et al. [1] includes an HR track of a one solar mass star with a metallicity $z=0.01$ in its Figure 13. Our HR diagram for $z=0.01$ is shown in Figure 3 .

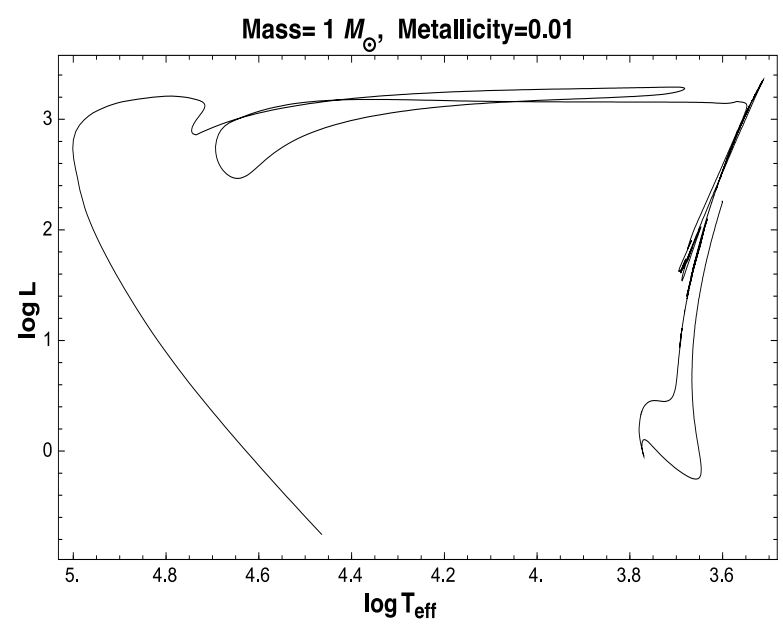

Figure 3. HR diagram of track from our simulation of a 1 solar mass star with $z=0.01$.

Using the pp_cno_extras_o18_ne22 reaction network, our one solar mass, $\bar{z}=0.01$ track exhibited a thermal pulse in the horizontal branch unlike reference [1] which did not include ${ }^{18} \mathrm{O}$ and ${ }^{22} \mathrm{Ne}$ in the simulation used for that diagram (Figure 13 of reference [1]). However in the same HR diagram of reference [1], a 1.25 solar mass, $\mathrm{z}=0.01$ track did exhibit a thermal pulse in the horizontal branch. These differences between tracks of simulations using different masses, metallicities, or reaction networks show the sensitivity of the simulations to initial conditions.

We would like to thank the MESA team for making the MESA package openly available. The MESA package can be found at mesa.sourceforge.net .

\section{References}

1. B. Paxton et al., ApJS 192, 3 (2011)

2. J. Hurley et al., Mon. Not. R. Astron. Soc. 315, 543 (2000)

3. M. Asplund and K. Lind, Proc. IAU Symp. No. 268 (2010) 